\title{
APPROXIMATION OF FIXED POINTS OF ASYMPTOTICALLY NONEXPANSIVE MAPPINGS
}

\author{
JÜRGEN SCHU
}

(Communicated by Palle E. T. Jorgensen)

\begin{abstract}
Let $T$ be an asymptotically nonexpansive self-mapping of a nonempty closed, bounded, and starshaped (with respect to zero) subset of a smooth reflexive Banach space possessing a duality mapping that is weakly sequentially continuous at zero. Then, if id $-T$ is demiclosed and $T$ satisfies a strengthened regularity condition, the iteration process $z_{n+1}:=\mu_{n+1} T^{n}\left(z_{n}\right)$ converges strongly to some fixed point of $T$, provided $\left(\mu_{n}\right)$ has certain properties.
\end{abstract}

\section{INTRODUCTION}

Motivated by the papers of B. Halpern [4] and P. Vijayaraju [7], we deal with the convergence of almost fixed points $x_{n}=\mu_{n} T^{n}\left(x_{n}\right)$ of an asymptotically nonexpansive mapping $T$. This class of functions was introduced by $\mathrm{K}$. Goebel and W. A. Kirk [3] in 1972. In $\S 1$, convergence of the sequence $\left(x_{n}\right)$ to some fixed point of $T$ is shown, assuming that $T$ is uniformly asymptotically regular and id $-T$ is demiclosed. These assumptions have been made by P. Vijayaraju [7] to ensure the existence of a fixed point of $T$.

By strengthening the regularity condition on $T$ in $\S 2$, we establish the convergence of the explicit iteration scheme $z_{n+1}:=\mu_{n+1} T^{n}\left(z_{n}\right)$ to some fixed point of $T$. This iteration method is similar to one that was introduced by $B$. Halpern [4].

\section{CONVERGENCE OF THE SEQUENCE OF APPROXIMATE FIXED POINTS}

We shall begin by recalling some definitions needed in the sequel.

Definition 1.1. Let $(E,\|\cdot\|)$ be a normed space, $\varnothing \neq A \subset E ; \mu: \mathbb{R}^{+} \rightarrow \mathbb{R}^{+}$; $J: E \rightarrow E^{*}$; and $x_{0} \in E$.

(1) $(E,\|\cdot\|)$ is called smooth: $\Longleftrightarrow\|\cdot\|$ is Gateaux-differentiable on $\partial \bar{B}(0,1)$.

(2) $\mu$ is said to be a gauge function: $\Longleftrightarrow \mu$ is continuous and strictly increasing with $\mu(0)=0$ and $\lim _{x \rightarrow \infty} \mu(x)=\infty$.

Received by the editors November 12, 1989 and, in revised form, February 20, 1990.

1980 Mathematics Subject Classification (1985 Revision). Primary 47H10; Secondary 40A05, $47 \mathrm{H} 09$.

Key words and phrases. Fixed point, iteration process, asymptotically nonexpansive mapping. 
(3) The set-valued duality mapping associated with $\mu$ is given by $J_{E}^{\mu}(x):=$ $\left\{u \in E^{*} \mid u(x)=\|u\|\|x\|\right.$ and $\left.\|u\|=\mu(\|x\|)\right\}$ and $J$ is called a duality mapping with respect to $\mu: \Longleftrightarrow J(x) \in J_{E}^{\mu}(x)$ for all $x \in E$. For abbreviation, we set $J_{E}:=J_{E}^{\text {id }}$.

(4) $J$ is weakly sequentially continuous at $x_{0}$ : $\Longleftrightarrow$ for all $\left(x_{n}\right) \in E^{\mathbb{N}}$ with $\left(x_{n}\right) \rightarrow x_{0}$, it follows that $\left(J\left(x_{n}\right)\right) \stackrel{*}{\rightarrow} J\left(x_{0}\right)$.

Remark. It is well known that $J_{E}^{\mu}$ is single-valued if and only if $(E,\|\cdot\|)$ is smooth (see, e.g., [1]). In this case, we regard $J_{E}^{\mu}$ as a mapping from $E$ to $E^{*}$. In all our proofs we assume, without loss of generality, that $J_{E}^{\mu}$ is normalized, i.e., $\mu=\mathrm{id}$. Futhermore, in the sequel all normed spaces are assumed to be real Banach spaces.

Definition 1.2. Let $(E,\|\cdot\|)$ be a normed space; $\varnothing \neq A \subset E ; T: A \rightarrow A$; $\left(k_{n}\right) \in[1, \infty)^{\mathbb{N}}$.

(1) $T$ is said to be asymptotically nonexpansive with sequence $\left(k_{n}\right)$ : $\Longleftrightarrow$ $\lim \left(k_{n}\right)=1$ and $\left\|T^{n}(x)-T^{n}(y)\right\| \leq k_{n}\|x-y\|$ for all $n \in \mathbb{N}$ and all $x, y \in A$.

(2) $T$ is called demiclosed: $\Longleftrightarrow$ for all $\left(x_{n}\right) \in A^{\mathbb{N}}$ and all $x, y \in E$ with $\left(x_{n}\right) \rightarrow x$ and $\lim \left(T\left(x_{n}\right)\right)=y$, it follows that $x \in A$ and $T(x)=y$.

(3) $T$ is called uniformly asymptotically regular: $\Longleftrightarrow$ for each $\varepsilon>0$ there is $n_{0} \in \mathbb{N}$, such that $\left\|T^{n}(x)-T^{n+1}(x)\right\| \leq \varepsilon$ for all $n \geq n_{0}$ and all $x \in A$.

Lemma 1.3. Let $(E,\|\cdot\|)$ be a smooth normed space; $\varnothing \neq A \subset E ; T: A \rightarrow A$ asymptotically nonexpansive with sequence $\left(k_{n}\right) \in[1, \infty)^{\mathbb{N}} ; x, y \in A ; \lambda \in$ $(0,1) ; n \in \mathbb{N} ; x=\lambda T^{n}(x) ;$ and $y=T^{n}(y)$. Then

$$
J_{E}(y-x)(x) \geq-\frac{k_{n}-1}{1 / \lambda-1}\|x-y\|^{2} .
$$

Proof. Set $\alpha:=\frac{1}{\lambda}-1>0, z:=\alpha x$, and $w:=y-x$. Then

$$
\begin{aligned}
\|z-w\| & =\|(1+\alpha) x-y\|=\left\|\frac{1}{\lambda} x-y\right\| \\
& =\left\|T^{n}(x)-T^{n}(y)\right\| \leq k_{n}\|x-y\|=k_{n}\|w\| .
\end{aligned}
$$

Hence

$$
\begin{aligned}
J_{E}(y-x)(x) & =\frac{1}{\alpha} J_{E}(w)(z)=\frac{1}{\alpha} J_{E}(w)(z-w)+\frac{1}{\alpha} J_{E}(w)(w) \\
& \geq-\frac{1}{\alpha}\|w\|\|z-w\|+\frac{1}{\alpha}\|w\|^{2} \geq \frac{1}{\alpha}\left(1-k_{n}\right)\|w\|^{2} \\
& =\left(\left(1-k_{n}\right) /\left(\left(\frac{1}{\lambda}\right)-1\right)\right)\|x-y\|^{2} .
\end{aligned}
$$

The following lemma can be verified by an easy calculation.

Lemma 1.4. Let $\lambda \in\left(\frac{1}{2}, 1\right)$. Then

(1) $\lambda^{2} /(2 \lambda-1)>1$, and

(2) $(\lambda k-\lambda) /(k-\lambda) \leq 1-\lambda$ for all $k \in\left[1, \lambda^{2} /(2 \lambda-1)\right]$. 
Remark. Part (1) of Lemma 1.4 shows that the assumption $k_{n} \leq \lambda_{n}^{2} /\left(2 \lambda_{n}-1\right)$ and $k_{n} \in[1, \infty)$ of our next lemma makes sense.

Lemma 1.5. Let $(E,\|\cdot\|)$ be a smooth normed space possessing a duality mapping $J: E \rightarrow E^{*}$ that is weakly sequentially continuous at $0 ; \varnothing \neq A \subset$ $E$ bounded; $T: A \rightarrow A$ asymptotically nonexpansive with sequence $\left(k_{n}\right) \in$ $[1, \infty)^{\mathbb{N}} ; \quad\left(x_{n}\right) \in A^{\mathbb{N}} ; x \in A ;\left(x_{\varphi_{n}}\right)$ a subsequence of $\left(x_{n}\right)$ with $\left(x_{\varphi_{n}}\right)-x ;$ $\left(\lambda_{n}\right) \in\left(\frac{1}{2}, 1\right)^{\mathbb{N}}$ with $\lim \left(\lambda_{n}\right)=1 ; x=T(x) ; x_{n}=\left(\lambda_{n} / k_{n}\right) T^{n}\left(x_{n}\right)$ for all $n \in \mathbb{N}$; and $k_{n} \leq \lambda_{n}^{2} /\left(2 \lambda_{n}-1\right)$ for all $n \in \mathbb{N}$. Then

(1) $\lim \left(x_{\varphi_{n}}\right)=x$, and

(2) $J(y-x)(x) \geq 0$ for all $y \in \operatorname{Fix}(T)$.

$(\operatorname{Fix}(T):=\{z \in A \mid T(z)=z\})$

Proof. (1) Since $\left(\lambda_{n} / k_{n}\right) \in(0,1)^{\mathrm{N}}$, it follows from Lemma 1.3 that

$$
J\left(x-x_{n}\right)\left(x_{n}\right) \geq-\frac{k_{n}-1}{k_{n} / \lambda_{n}-1}\left\|x_{n}-x\right\|^{2} \quad \text { for all } n \in \mathbb{N} .
$$

We may choose $M>0$ such that $\left\|x_{n}-z\right\|^{2} \leq M$ for all $n \in \mathbb{N}$ and all $z \in A$ because of the boundedness of $A$. Obviously, $\left(k_{n}-1\right) /\left(k_{n} / \lambda_{n}-1\right) \geq 0$ and from Lemma 1.4 we know, that

$$
\frac{k_{n}-1}{k_{n} / \lambda_{n}-1}=\frac{\lambda_{n} k_{n}-\lambda_{n}}{k_{n}-\lambda_{n}} \leq 1-\lambda_{n}
$$

Hence $J\left(x-x_{n}\right)\left(x_{n}\right) \geq-\left(1-\lambda_{n}\right) M$, and therefore, for all $n \in \mathbb{N}$,

$$
\left\|x-x_{n}\right\|^{2}=J\left(x-x_{n}\right)(x)-J\left(x-x_{n}\right)\left(x_{n}\right) \leq J\left(x-x_{n}\right)(x)+\left(1-\lambda_{n}\right) M,
$$

where

(a) $\lim \left(1-\lambda_{n}\right)=0$,

(b) $\left(x_{\varphi_{n}}-x\right) \rightarrow 0$, hence $\lim J\left(x-x_{\varphi_{n}}\right)(x)=0$.

Thus we conclude that $\lim \left\|x-x_{\varphi_{n}}\right\|=0$.

(2) Fix $y \in \operatorname{Fix}(T)$. As already shown in (1), for $y=T(y)$ instead of $x$, it follows that

$$
J\left(y-x_{n}\right)\left(x_{n}\right) \geq-\left(1-\lambda_{n}\right) M \text { for all } n \in \mathbb{N} .
$$

Since $(E,\|\cdot\|)$ is smooth, $J$ is strong-weak ${ }^{(*)}$ continuous (see e.g., [2]), and so from $\lim \left(y-x_{\varphi_{n}}\right)=y-x$, we conclude that $\left(J\left(y-x_{\varphi_{n}}\right)\right) \stackrel{*}{-} J(y-x)$. Hence $\lim J\left(y-x_{\varphi_{n}}\right)\left(x_{\varphi_{n}}\right)^{n}=J(y-x)(x)$. This, together with $(*)$ and $\lim \left(1-\lambda_{n}\right)=0$, yields $J(y-x)(x) \geq 0$.

Remark. Lemmas 1.3 and 1.5 are similar to some related results for nonexpansive mappings; namely, [6, Lemmas 4, 5]. The following theorem was demonstrated by P. Vijayaraju in the course of the proof of [7, Theorems 2.1, 2.2]. 
Theorem 1.6. Let $(E,\|\cdot\|)$ be a Banach space; $\varnothing \neq A \subset E$ closed, bounded, and starshaped with respect to $0 ; T: A \rightarrow A$ asymptotically nonexpansive with sequence $\left(k_{n}\right) \in[1, \infty)^{\mathbb{N}}$ and uniformly asymptotically regular, $\left(\lambda_{n}\right) \in[0,1)^{\mathbb{N}}$; and $\lim \left(\lambda_{n}\right)=1$. Then

(1) for each $n \in \mathbb{N}$ there is exactly one $x_{n} \in A$ such that $x_{n}=\left(\lambda_{n} / k_{n}\right) T^{n}\left(x_{n}\right)$, and

(2) $\lim \left\|x_{n}-T\left(x_{n}\right)\right\|=0$.

Now we are able to prove the main result of this section.

Theorem 1.7. Let $(E,\|\cdot\|)$ be a smooth reflexive Banach space possessing a duality mapping $J: E \rightarrow E^{*}$ that is weakly sequentially continuous at $0 ; \varnothing \neq A \subset E$ closed, bounded, and starshaped with respect to $0 ; T: A \rightarrow A$ asymptotically nonexpansive with sequence $\left(k_{n}\right) \in[1, \infty)^{\mathbb{N}}$ and uniformly asymptotically regular, id $-T$ demiclosed; $\left(\lambda_{n}\right) \in\left(\frac{1}{2}, 1\right)^{\mathbb{N}} ; \lim \left(\lambda_{n}\right)=1 ; k_{n} \leq \lambda_{n}^{2} /\left(2 \lambda_{n}-1\right)$ for all $n \in \mathbb{N}$. Then

(1) for each $n \in \mathbb{N}$ there is exactly one $x_{n} \in A$ such that $x_{n}=\left(\lambda_{n} / k_{n}\right) T^{n}\left(x_{n}\right)$, and

(2) $\left(x_{n}\right)$ converges strongly to some fixed point of $T$.

Proof. Part (1) follows from Theorem 1.6, so that it remains to prove part (2). Since $(E,\|\cdot\|)$ is reflexive and $A$ is bounded, there exists $z \in E$ and a subsequence $\left(x_{\mu_{n}}\right)$ of $\left(x_{n}\right)$ such that $\left(x_{\mu_{u}}\right) \rightarrow z$ (Pettis' theorem). From Theorem 1.6, $\lim \left\|x_{n}-T\left(x_{n}\right)\right\|=0$, so from the demiclosedness of id $-T$, $z \in A$ and $T(z)=z$.

Applying Lemma 1.5, we get

$$
J(y-z)(z) \geq 0 \text { for all } y \in \operatorname{Fix}(T) .
$$

Fix $\varphi: \mathbb{N} \rightarrow \mathbb{N}$ and injective.

Repeating the above argument, we find a subsequence $\left(x_{\varphi_{\psi_{n}}}\right)$ of $\left(x_{\varphi_{n}}\right)$ converging weakly to some $x \in A$ such that $x=T(x)$ and

$$
J(y-x)(x) \geq 0 \text { for all } y \in \operatorname{Fix}(T) .
$$

Substituting $x$ into $\left(^{*}\right)$ and $z$ into $\left(^{* *}\right)$, it follows that $J(x-z)(z) \geq 0$ and $J(z-x)(x) \geq 0$. Hence $0 \leq J(x-z)(-x)+J(x-z)(z)=-\|x-z\|^{2} \leq 0$, so that $x=z$, and therefore $\left(x_{\varphi_{\psi_{n}}}\right)-z$. This shows that $\left(x_{n}\right)-z$, and we now apply Lemma 1.5 (with $\varphi:=\mathrm{id}$ ) to obtain $\lim \left\|x_{n}-z\right\|=0$.

Remark. For $\left(k_{n}\right) \in(1, \infty)^{\mathbb{N}}$ with $\lim \left(k_{n}\right)=1$, the conditions $\left(\lambda_{n}\right) \in\left(\frac{1}{2}, 1\right)^{\mathbb{N}}$, $\lim \left(\lambda_{n}\right)=1$, and $k_{n} \leq \lambda_{n}^{2} /\left(2 \lambda_{n}-1\right)$ for all $n \in \mathbb{N}$ are fulfilled, for example, if one chooses $\lambda_{n}:=k_{n}-\sqrt{k_{n}^{2}-k_{n}}$ for all $n \in \mathbb{N}$.

\section{AN EXPLICIT ITERATION SCHEME}

Now we give an explicit iteration scheme for the approximation of a fixed point of $T$. In analogy to B. Halpern's work [4], we use the fact that the 
implicit iteration process $x_{n}=\left(\lambda_{n} / k_{n}\right) T^{n}\left(x_{n}\right)$ already converges to some fixed point of $T$ to show that the same holds for the explicit iteration procedure $z_{n+1}:=\left(\lambda_{n+1} / k_{n+1}\right) T^{n}\left(z_{n}\right)$

In addition to several technical assumptions on the values $\lambda_{n}$ and $k_{n}$, we require that $T$ fulfills a certain strengthened regularity condition (assumption (b) of Theorem 2.2). Although parts of the proof of Theorem 2.2 are parallel to those of the proof of [4, Theorem 3], they are included for the sake of completeness.

Definition 2.1. Let $\left(\varepsilon_{n}\right) \in(0, \infty)^{\mathbb{N}}$ and $\left(\mu_{n}\right) \in(0,1)^{\mathbb{N}} \cdot\left(\left(\varepsilon_{n}\right),\left(\mu_{n}\right)\right)$ is called admissible : $\Longleftrightarrow$

(1) $\left(\varepsilon_{n}\right)$ is decreasing;

(2) $\left(\mu_{n}\right)$ is strictly increasing with $\lim \left(\mu_{n}\right)=1$;

(3) there exists $\left(\beta_{n}\right) \in \mathbb{N}^{\mathbb{N}}$ such that

(i) $\left(\beta_{n}\right)$ is increasing,

(ii) $\lim \left(\beta_{n}\left(1-\mu_{n}\right)\right)=\infty$,

(iii) $\lim \left(\left(1-\mu_{n+\beta_{n}}\right) /\left(1-\mu_{n}\right)\right)=1$, and

(iv) $\lim \left(\left(\varepsilon_{n} \beta_{n} \mu_{n+\beta_{n}}\right) /\left(1-\mu_{n}\right)\right)=0$.

Remark. (a) Parts (2) and (3) (i)-(iii) are due to B. Halpern [4]. (b) $\lim \left(\beta_{n}\right)=$ $\infty$ because of (3)(ii) and (2), so that it follows from (3)(iv) that $\lim \left(\varepsilon_{n}\right)=0$.

Theorem 2.2. Let $(E,\|\cdot\|)$ be a normed space; $\varnothing \neq A \subset E$ bounded and starshaped with respect to $0 ; T: A \rightarrow A$ asymptotically nonexpansive with sequence $\left(k_{n}\right) \in[1, \infty)^{\mathbb{N}} ; \quad\left(\lambda_{n}\right) \in(0,1)^{\mathbb{N}}$ with $\lim \left(\lambda_{n}\right)=1 ;\left(x_{n}\right) \in A^{\mathbb{N}} ;$ $\mu_{n}:=\lambda_{n} / k_{n}$ for all $n \in \mathbb{N} ; \quad z_{0} \in A ; x_{n}=\left(\lambda_{n} / k_{n}\right) T^{n}\left(x_{n}\right)$ for all $n \in \mathbb{N}$, and $z_{n+1}:=\left(\lambda_{n+1} / k_{n+1}\right) T^{n}\left(z_{n}\right)$ for all $n \in \mathbb{N}_{0} ;\left(\varepsilon_{n}\right) \in(0, \infty)^{\mathbb{N}}$ such that $\left(\left(\varepsilon_{n}\right),\left(\mu_{n}\right)\right)$ is admissible;

(a) $\left(\left(1-\mu_{n}\right) /\left(1-\lambda_{n}\right)\right)$ bounded;

(b) $\left\|T^{n}(x)-T^{n+1}(x)\right\| \leq \varepsilon_{n}$ for all $n \in \mathbb{N}$ and all $x \in A$.

Assume further that $q:=\lim \left(x_{n}\right)$ exists. Then $\lim \left(z_{n}\right)=q$.

Proof. Observe that $\left(z_{n}\right) \in A^{\mathbb{N}}$ is well defined, because $T(A) \subset A,\left(\mu_{n}\right) \in$ $(0,1)^{\mathbb{N}}$, and $A$ is starshaped with respect to 0 . Since $A$ is bounded and $T$ is Lipschitzian, there is a $d>0$ such that $\|z\| \leq d$ for all $z \in A$ and there is a $c>0$ such that $\|T(w)\| \leq c$ for all $w \in A$. Additionally, (a) implies the existence of a number $\kappa>0$, such that $\left(1-\mu_{n}\right) /\left(1-\lambda_{n}\right) \leq \kappa$ for all $n \in \mathbb{N}$, and we may choose $\left(\beta_{n}\right)$ according to Definition 2.1. For $n>p \geq i$, we have

$$
\left\|z_{p+1}-x_{i}\right\| \leq\left\|\mu_{p+1} T^{p}\left(z_{p}\right)-\mu_{i} T^{i}\left(z_{p}\right)\right\|+\left\|\mu_{i} T^{i}\left(z_{p}\right)-\mu_{i} T^{i}\left(x_{i}\right)\right\|,
$$

where

$$
\left\|\mu_{i} T^{i}\left(z_{p}\right)-\mu_{i} T^{i}\left(x_{i}\right)\right\| \leq \mu_{i} k_{i}\left\|z_{p}-x_{i}\right\|=\lambda_{i}\left\|z_{p}-x_{i}\right\|,
$$


and

$$
\begin{aligned}
& \left\|\mu_{p+1} T^{p}\left(z_{p}\right)-\mu_{i} T^{i}\left(z_{p}\right)\right\| \\
& \quad \leq\left\|\mu_{p+1} T^{p}\left(z_{p}\right)-\mu_{p+1} T^{i}\left(z_{p}\right)\right\|+\left\|\mu_{p+1} T^{i}\left(z_{p}\right)-\mu_{i} T^{i}\left(z_{p}\right)\right\| \\
& \quad \leq \mu_{p+1}\left\|T^{p}\left(z_{p}\right)-T^{i}\left(z_{p}\right)\right\|+\left|\mu_{p+1}-\mu_{i}\right| c .
\end{aligned}
$$

Since $n>p \geq i$, it follows that $\left|\mu_{p+1}-\mu_{i}\right| \leq \mu_{n}-\mu_{i}$. Additionally,

$$
\begin{aligned}
\left\|T^{p}\left(z_{p}\right)-T^{i}\left(z_{p}\right)\right\| & \leq \sum_{j=i}^{p-1}\left\|T^{j+1}\left(z_{p}\right)-T^{j}\left(z_{p}\right)\right\| \\
& \leq \sum_{j=i}^{p-1} \varepsilon_{j} \leq \sum_{j=i}^{p-1} \varepsilon_{i}=(p-i) \varepsilon_{i} \leq(n-i) \varepsilon_{i} .
\end{aligned}
$$

Combining the above inequalities, we get

(1) $\left\|z_{p+1}-x_{i}\right\| \leq \lambda_{i}\left\|z_{p}-x_{i}\right\|+\left(\mu_{n}-\mu_{i}\right) c+\mu_{n}(n-i) \varepsilon_{i}$ for all $n>p \geq i$,

from which it follows by induction that

$$
\left\|z_{m+j}-x_{i}\right\| \leq \lambda_{i}^{j}\left\|z_{m}-x_{i}\right\|+\left(\mu_{n}-\mu_{i}\right) c \sum_{\nu=0}^{j-1} \lambda_{i}^{\nu}+\mu_{n}(n-i) \varepsilon_{i} \sum_{\nu=0}^{j-1} \lambda_{i}^{\nu},
$$

for all $n>m \geq i$ and $j \leq n-m$. Since $\sum_{\nu=0}^{j-1} \lambda_{i}^{\nu} \leq 1 /\left(1-\lambda_{i}\right)$, we obtain, defining $j=n-m$,

(2) $\left\|z_{n}-x_{i}\right\| \leq \lambda_{i}^{n-m}\left\|z_{m}-x_{i}\right\|+\left(\mu_{n}-\mu_{i}\right)\left(1-\lambda_{i}\right)^{-1} c+\mu_{n}(n-i) \varepsilon_{i}\left(1-\lambda_{i}\right)^{-1}$

for all $n>m \geq i$.

Set $m:=i$ and $n:=i+\beta_{i}>m$ to get

$$
\left\|z_{i+\beta_{i}}-x_{i}\right\| \leq 2 d \lambda_{i}^{\beta_{i}}+\left(\mu_{i+\beta_{i}}-\mu_{i}\right)\left(1-\lambda_{i}\right)^{-1} c+\mu_{i+\beta_{i}} \beta_{i} \varepsilon_{i}\left(1-\lambda_{i}\right)^{-1},
$$

for all $i \in \mathbb{N}$. Observe that

(i) $\left|\mu_{i+\beta_{i}}-\mu_{i}\right|\left|1-\lambda_{i}\right|^{-1}=\left|1-\left(1-\mu_{i+\beta_{i}}\right)\left(1-\mu_{i}\right)^{-1}\right|\left|\left(1-\mu_{i}\right) /\left(1-\lambda_{i}\right)\right|$ $\leq \kappa\left|1-\left(1-\mu_{i+\beta_{i}}\right)\left(1-\mu_{i}\right)^{-1}\right|$,

from which we conclude that $\lim \left(\left(\mu_{i+\beta_{i}}-\mu_{i}\right) /\left(1-\lambda_{i}\right)\right)=0$, taking into account the admissibility of $\left(\left(\varepsilon_{n}\right),\left(\mu_{n}\right)\right)$. Also,

(ii) $\log \left(\lambda_{i}^{\beta_{i}}\right) \leq \beta_{i}\left(\lambda_{i}-1\right) \leq-\beta_{i}\left(1-\mu_{i}\right) 1 / \kappa$. Since $\left(\left(\varepsilon_{n}\right),\left(\mu_{n}\right)\right)$ is admissible, it follows that $\lim \left(\log \left(\lambda_{i}^{\beta_{i}}\right)\right)=-\infty$, and thus $\lim \left(\lambda_{i}^{\beta_{i}}\right)=0$. In addition,

(iii) $0 \leq \mu_{i+\beta_{i}} \beta_{i} \varepsilon_{i}\left(1-\lambda_{i}\right)^{-1} \leq \kappa \mu_{i+\beta_{i}} \beta_{i} \varepsilon_{i}\left(1-\mu_{i}\right)^{-1}$, from which it follows that $\lim \left(\left(\mu_{i+\beta_{i}} \beta_{i} \varepsilon_{i}\right) /\left(1-\lambda_{i}\right)\right)=0$, again using the admissibility of $\left(\left(\varepsilon_{n}\right),\left(\mu_{n}\right)\right)$. Hence

$$
\lim \left\|z_{i+\beta_{i}}-x_{i}\right\|=0
$$


As already noticed in the proof of [4, Theorem 3], there exists $j: \mathbb{N} \rightarrow \mathbb{N}$ and $n_{0} \in \mathbb{N}$ such that $\lim \left(j_{n}\right)=\infty$ and $j_{n}+\beta_{j_{h}}<n \leq j_{n}+1+\beta_{j_{n}+1}$ for all $n \geq n_{0}$. Now let $n \geq n_{0}$. With $n>m:=j_{n}+\beta_{j_{n}} \geq i:=j_{n}+1$, it follows from (2) that

$$
\begin{aligned}
\left\|z_{n}-x_{j_{n}+1}\right\| \leq & \left\|z_{\left(j_{n}+\beta_{j n}\right)}-x_{j_{n}+1}\right\|+\left(\mu_{n}-\mu_{j_{n}+1}\right)\left(1-\lambda_{j_{n}+1}\right)^{-1} c \\
& +\mu_{n}\left(n-\left(j_{n}+1\right)\right) \varepsilon_{j_{n}+1}\left(1-\lambda_{j_{n}+1}\right)^{-1} \text { for all } n \in \mathbb{N},
\end{aligned}
$$

where

(i) $0 \leq\left(\mu_{n}-\mu_{j_{n}+1}\right)\left(1-\lambda_{j_{n}+1}\right)^{-1} \leq\left(\mu_{\left(j_{n}+1+\beta_{j_{n}+1}\right)}-\mu_{j_{n}+1}\right)\left(1-\lambda_{j_{n}+1}\right)^{-1}$, which tends to 0 by $2(\mathrm{i})$.

(ii) $0 \leq \mu_{n}\left(n-\left(j_{n}+1\right)\right) \varepsilon_{j_{n}+1}\left(1-\lambda_{j_{n}+1}\right)^{-1} \leq \mu_{\left(j_{n}+1+\beta_{j_{n}+1}\right)} \beta_{j_{n}+1} \varepsilon_{j_{n}+1}\left(1-\lambda_{j_{n}+1}\right)^{-1}$, which tends to 0 by (2)(iii).

(iii) $\left\|z_{\left(j_{n}+\beta_{j_{n}}\right)}-x_{j_{n}+1}\right\| \leq\left\|z_{\left(j_{n}+\beta_{j_{n}}\right)}-x_{j_{n}}\right\|+\left\|x_{j_{n}}-q\right\|+\left\|q-x_{j_{n}+1}\right\|$, which also tends to 0 because $\lim \left\|x_{n}-q\right\|=0$ and $\lim \left\|z_{\left(j_{n}+\beta_{j_{n}}\right)}-x_{j_{n}}\right\|=0$ by (3). Hence $\lim \left\|z_{n}-x_{j_{n}+1}\right\|=0$. Finally, since $\left\|z_{n}-q\right\| \leq\left\|z_{n}-x_{j_{n}+1}\right\|+\left\|x_{j_{n}+1}-q\right\|$, it follows that $\lim \left\|z_{n}-q\right\|=0$.

We now prove the main result of this section.

Theorem 2.3. Let $(E,\|\cdot\|)$ be a reflexive smooth Banach space possessing a duality mapping that is weakly sequentially continuous at $0 ; \varnothing \neq A \subset E$ closed, bounded, and starshaped with respect to $0 ; T: A \rightarrow A$ asymptotically nonexpansive with sequence $\left(k_{n}\right) \in[1, \infty)^{\mathbb{N}} ;$ id $-T$ demiclosed; $\left(\lambda_{n}\right) \in\left(\frac{1}{2}, 1\right)^{\mathbb{N}}$; $z_{0} \in A ; \quad z_{n+1}:=\left(\lambda_{n+1} / k_{n+1}\right) T^{n}\left(z_{n}\right)$ for all $n \in \mathbb{N}_{0}$;

(a) $\lim \left(\lambda_{n}\right)=1 ; \mu_{n}:=\lambda_{n} / k_{n}$ for all $n \in \mathbb{N} ; k_{n} \leq \lambda_{n}^{2} /\left(2 \lambda_{n}-1\right)$ for all $n \in$ $\mathbb{N} ;\left(\left(1-\mu_{n}\right) /\left(1-\lambda_{n}\right)\right)$ bounded $;\left(\varepsilon_{n}\right) \in(0, \infty)^{\mathbb{N}}$, such that $\left(\left(\varepsilon_{n}\right),\left(\mu_{n}\right)\right)$ is admissible;

(b) $\left\|T^{n}(x)-T^{n+1}(x)\right\| \leq \varepsilon_{n}$ for all $n \in \mathbb{N}$ and all $x \in A$.

Then $\left(z_{n}\right)$ converges strongly to some fixed point of $T$.

Proof. Since $\left(\left(\varepsilon_{n}\right),\left(\mu_{n}\right)\right)$ is admissible, it follows that $\lim \left(\varepsilon_{n}\right)=0$, so that assumption (b) implies that $T$ is uniformly asymptotically regular. The result follows by a combination of Theorems 1.7, 2.2.

Remark. (1) For a related result concerning the weak convergence of the successive approximations $x_{n+1}:=T\left(x_{n}\right)$ to some fixed point of an asymptotically nonexpansive mapping $T$ in an Opial space we refer the reader to [5].

(2) Theorem 2.3, of course, is applicable to nonexpansive mappings as well (set $k_{n}:=1$ for all $n \in \mathbb{N}$ ). In this special case, however, there is a much better result: [6, Theorem 10] allows us to drop the assumptions that id $-T$ is demiclosed and that $\left\|T^{n}(x)-T^{n+1}(x)\right\| \leq \varepsilon_{n}$ for all $n \in \mathbb{N}$ and all $x \in A$, provided use is made of the iteration scheme $z_{n+1}:=\lambda_{n+1} T\left(z_{n}\right), T$ is nonexpansive, and $(E,\|\cdot\|)$ possesses a duality mapping that is weakly sequentially continuous on the whole of $E$. 
We close this section with a special case of Theorem 2.3, which shows that it is really possible to fulfill the assumptions made on $\left(\lambda_{n}\right),\left(\mu_{n}\right),\left(\varepsilon_{n}\right)$, and $\left(k_{n}\right)$.

Theorem 2.4. Let $(E,\|\cdot\|)$ be a reflexive smooth Banach space possessing a duality mapping that is weakly sequentially continuous at $0 ; \varnothing \neq A \subset E$ closed, bounded, and starshaped with respect to $0 ; T: A \rightarrow A$ asymptotically nonexpansive with sequence $(1+1 /(\sqrt{n+1}-1))$; id $-T$ demiclosed; $\mu_{n}:=1-(n+1)^{-1 / 4}$ for all $n \in \mathbb{N} ; \quad z_{0} \in A ; \quad z_{n+1}:=\mu_{n+1} T^{n}\left(z_{n}\right)$ for all $n \in \mathbb{N}_{0} ;$ and

$$
\left\|T^{n}(x)-T^{n+1}(x)\right\| \leq(n+1)^{-7 / 8} \text { for all } n \in \mathbb{N} \text { and all } x \in A .
$$

Then $\left(z_{n}\right)$ converges strongly to some fixed point of $T$.

Proof. For $n \in \mathbb{N}$ define $k_{n}:=1+1 /(\sqrt{n+1}-1), \lambda_{n}:=k_{n}-\sqrt{k_{n}^{2}-k_{n}}$, $\varepsilon_{n}:=(n+1)^{-7 / 8}$, and $\beta_{n}:=\left[(n+1)^{1 / 2}\right]_{G}$ (cf. Definition 2.1), where $[x]_{G}:=$ $\max \left\{n \in \mathbb{N}_{0} \mid n \leq x\right\}$. Then easy calculations show that all the assumptions of Theorem 2.3 are fulfilled, especially that $\mu_{n}=\lambda_{n} / k_{n}$ for all $n \in \mathbb{N}$ and that $\left(\left(\varepsilon_{n}\right),\left(\mu_{n}\right)\right)$ is admissible.

Remark. For $x \in A$ and $n, m \in \mathbb{N}$ with $n>m$, the following holds:

$$
\left\|T^{n}(x)-T^{m}(x)\right\| \leq \sum_{j=m}^{n-1}\left\|T^{j+1}(x)-T^{j}(x)\right\| \leq \sum_{j=m}^{n-1}(j+1)^{-7 / 8} .
$$

Nevertheless, since $\sum_{j=1}^{\infty}(j+1)^{-7 / 8}=\infty,(*)$ does not imply that $\left(T^{n}(x)\right)$ is a Cauchy sequence (from which it would follow that $\left(T^{n}(x)\right.$ ) converges strongly to some fixed point of $T$ ).

\section{ACKNOWLEDGMENT}

The author is grateful to Professor J. Reinermann for his interest in this paper.

\section{REFERENCES}

1. B. Beauzamy, Introduction to Banach spaces and their geometry, Mathematics Studies, vol. 68, North-Holland, Amsterdam, 1982.

2. J. Diestel, Geometry of Banach spaces-selected topics; Lecture Notes in Math., vol. 485, Springer-Verlag, Berlin, Heidelberg, and New York, 1975.

3. K. Goebel and W. A. Kirk, A fixed point theorem for asymptotically nonexpansive mappings, Proc. Amer. Math. Soc. 35(1) (1972), 171-174.

4. B. Halpern, Fixed points of nonexpanding maps, Bull. Amer. Math. Soc. 73 (1967), 957-961.

5. S. K. Samanta, Fixed point theorems in a Banach space satisfying Opial's condition, J. Indian Math. Soc. 45 (1981), 251-258. 
6. J. Schu, Iterative approximation of fixed points of nonexpansive mappings with starshaped domain, Comment. Math. Univ. Carolinae (to appear).

7. P. Vijayaraju, Fixed point theorems for asymptotically nonexpansive mappings, Bull. Calcutta Math. Soc. 80 (1988), 133-136.

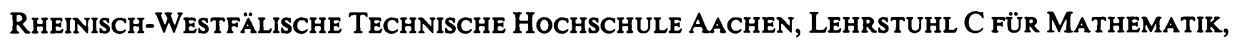
Templergraben 55, D-5100 Aachen, Federal RePublic of Germany 